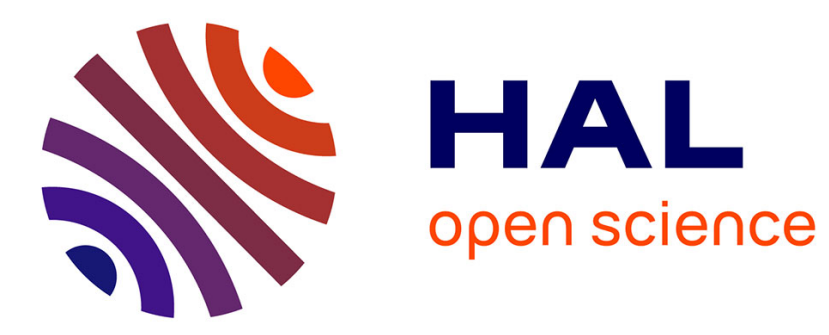

\title{
Migration des atomes de radon implantés dans les cristaux par énergie de recul
}

\author{
Guillaume Lambert, P. Bristeau
}

\section{To cite this version:}

Guillaume Lambert, P. Bristeau. Migration des atomes de radon implantés dans les cristaux par énergie de recul. Journal de Physique Colloques, 1973, 34 (C5), pp.C5-137-C5-138. 10.1051/jphyscol:1973526 . jpa-00215313

\section{HAL Id: jpa-00215313 https://hal.science/jpa-00215313}

Submitted on 1 Jan 1973

HAL is a multi-disciplinary open access archive for the deposit and dissemination of scientific research documents, whether they are published or not. The documents may come from teaching and research institutions in France or abroad, or from public or private research centers.
L'archive ouverte pluridisciplinaire HAL, est destinée au dépôt et à la diffusion de documents scientifiques de niveau recherche, publiés ou non, émanant des établissements d'enseignement et de recherche français ou étrangers, des laboratoires publics ou privés. 


\title{
MIGRATION DES ATOMES DE RADON IMPLANTÉS DANS LES CRISTAUX PAR ÉNERGIE DE RECUL
}

\author{
G. LAMBERT $(*)$, P. BRISTEAU \\ Centre des Faibles Radioactivités \\ Laboratoire mixte CNRS/CEA \\ 91190 Gif-sur-Yvette, France
}

\begin{abstract}
Résumé. - Des mesures radioactives simples permettent de montrer que la diffusion du radon à l'intérieur de monocristaux et de divers minéraux est très faible et que celle-ci ne peut expliquer le pouvoir émanateur des roches.
\end{abstract}

Abstract. - Simple radioactive measurements show that the diffusion of radon is very low within various monocrystals and that it cannot explain the emanating power of rocks.

1. Introduction. - La géochronologie radioactive repose sur l'hypothèse que les différents nuclides appartenant à une même chaîne de filiation radioactive restent stockés à l'intérieur du cristal mềme où ils ont été créés. Cette hypothèse a été mise en doute dans le cas des datations uranium/plomb, par suite de l'existence, en milieu de chaîne, d'un isotope du radon, donc d'un atome de gaz rare, a priori moins lié au cristal que les éléments métalliques. Par exemple :

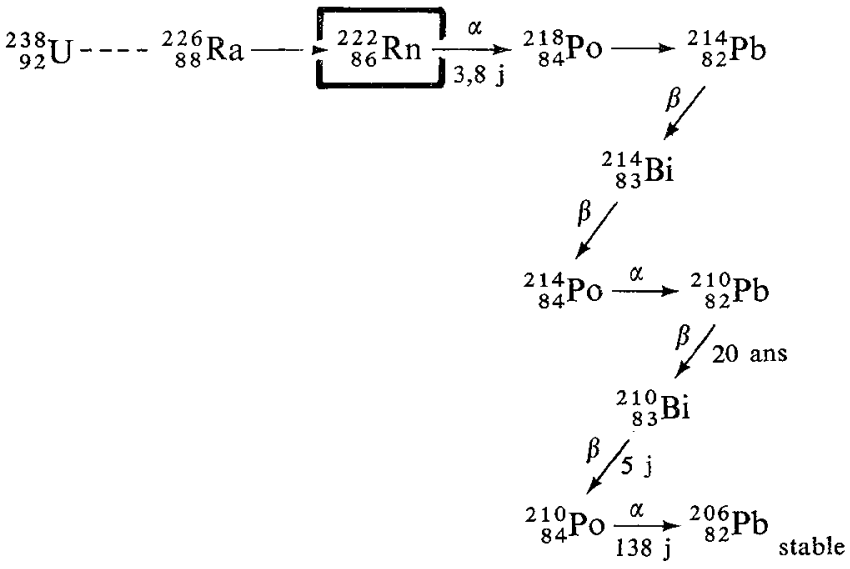

Nota: les périodes des descendants du radon qui ne sont pas indiquées sont inférieures à $20 \mathrm{~min}$.

Un élément radiogénique est susceptible de sortir du cristal de deux manières:

1. Par Énergie de Recul. - Celle-ci est négligeable dans le cas d'une réaction $\beta$, mais de l'ordre

(*) Professeur à l'Université de Picardie. de 100 à $200 \mathrm{keV}$ dans le cas d'une réaction $\alpha$, ce qui correspond à un parcours généralement inférieur à $500 \AA$.

Par suite de considérations géométriques simples, seuls sont alors affectés $25 \%$ des atomes produits dans la couche très mince de $500 \AA$, à la surface du cristal.

2. PAR DIFFUSION. - On doit d'abord remarquer que la vitesse de diffusion d'un atome de radon risque d'être affectée par les modifications du réseau cristallin dues à sa trace (il est produit par désintégration $\alpha$ du radium, donc avec une énergie de recul de $130 \mathrm{keV}$ ).

Les mesures de «pouvoir émanateur» de différents échantillons minéralogiques ont donné des résultats très contradictoires qui ne permettent pas de déterminer même l'ordre de grandeur du coefficient de diffusion du radon dans son cristal.

2. Principe des expériences. - Une face dressée de l'échantillon étudié est disposée dans le vide, à $1 \mathrm{~mm}$ environ d'une source mince de radium 226 (Fig. 1). Une partie des atomes de radon 222 produits sont ainsi directement implantés dans l'échantillon. L'irradiation dure au moins 2 à 10 périodes radioactives ( 1 à 5 semaines).

La face irradiée de l'échantillon est ensuite placée sous le détecteur d'un spectromètre $\alpha$, à l'intérieur d'une enceinte où l'on pompe constamment le radon qui pourrait se dégager. On mesure ainsi uniquement le rayonnement $\alpha$ du radon implanté et l'on compare la décroissance observée à la décroissance théorique du radon $(T=3,825$ j) (Fig. 2). 
I RRADIATION

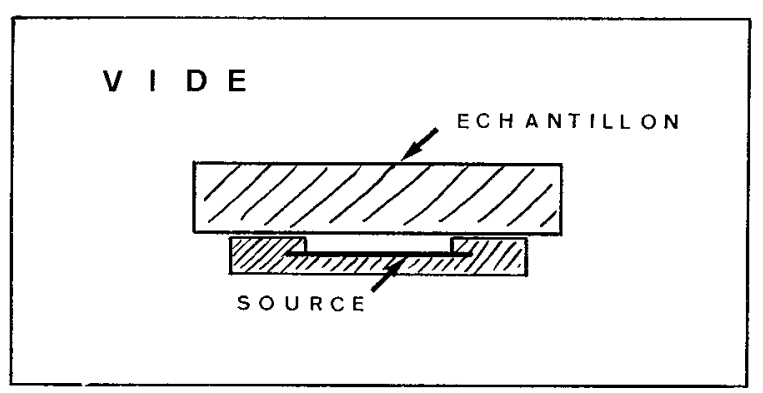

FIG. 1.

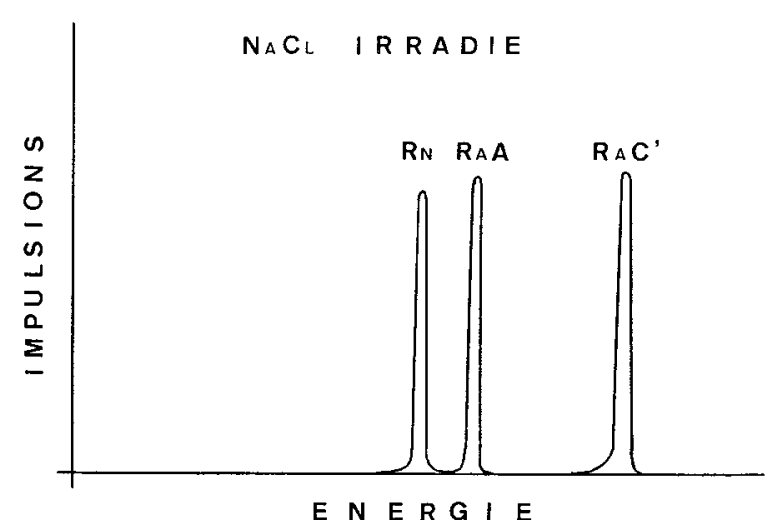

FIG. 2.

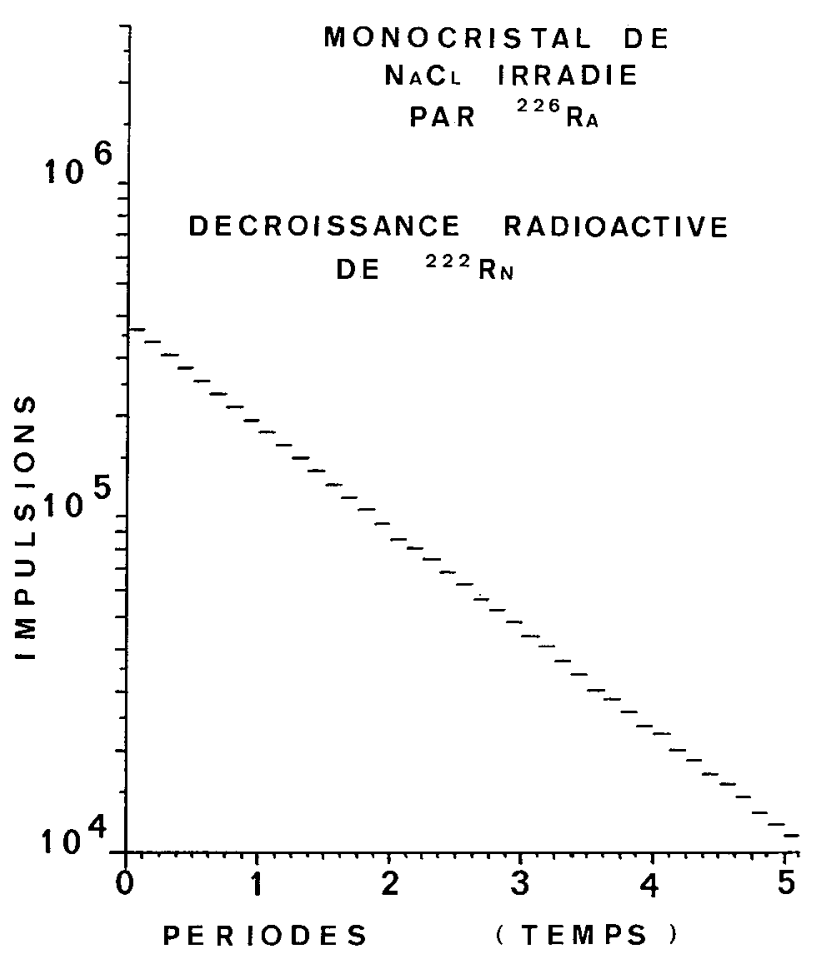

FIG. 3.
3. Résultats. - Quatre séries d'expériences ont été réalisées à ce jour :

Monocristal de $\mathrm{NaCl}$ - Température du laboPerte $1,7 \% \pm 0,7 \%$ (Fig. 3).

Mica clivé - Température du labo - Perte indécelable.

Calcaire dur $[1]-300^{\circ} \mathrm{C}-$ Perte $3,1 \% \pm 2,7 \%$ (Fig. 4).

Basalte [1] $-300^{\circ} \mathrm{C}-$ Perte entre 0 et $2 \%$.

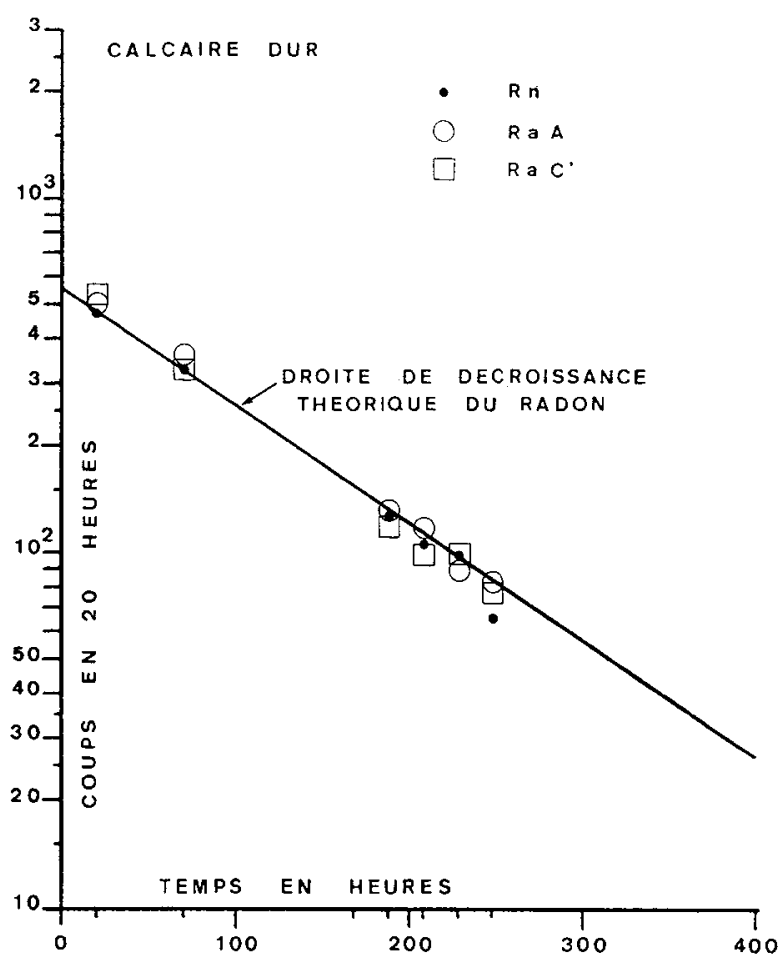

Frg. 4.

Compte tenu des profondeurs de pénétration du radon, on peut estimer que le coefficient de diffusion de ce nuclide dans un monocristal est, à la température ordinaire, compris entre $10^{-21}$ et $10^{-22} \mathrm{~cm}^{2} \cdot \mathrm{s}^{-1}$.

Les seules pertes de radon à considérer dans les cristaux d'un minéral naturel sont donc les pertes par recul, dont l'importance relative est d'autant plus faible que le grain cristallin est plus gros. Ce résultat, rassurant pour les datations $\mathrm{U} / \mathrm{Pb}$ et $\mathrm{Pb} 206 / \mathrm{Pb} 207$, pose le problème de la compréhension du pouvoir émanateur relativement élevé du sol en général.

Les auteurs remercient Mmes Taurel et Levelut qui leur ont aimablement procuré les monocristaux, ainsi que pour la préparation de la source de radium Mmes Legout et Ackaouy.

\section{Bibliographie}

[1] Lambert, G., Bristeau, P., Polian, G., C. R. Hebd. Séan. Acad. Sci., D Paris 274 (1972) 3333-3336. 